\title{
Inverse problems for mathematical models in social networks: from PDE to SDE
}

\author{
Sh. Zhang ${ }^{1 *}$, S. Kabanikhin ${ }^{2,3}$, O. Krivorotko ${ }^{2,3}$, Yu. Wang ${ }^{1}$ \\ ${ }^{1}$ Tianjin University of Finance and Economics, Tianjin, China \\ ${ }^{2}$ Institute of Computational Mathematics and Mathematical Geophysics SB RAS, Novosibirsk, Russia \\ ${ }^{3}$ Novosibirsk State University, Novosibirsk, Russia \\ *e-mail:shuhua55@126.com
}

Key words: inverse problems, mathematical modelling, partial differential equations, stochastic differential equations, social processes, optimization, Hamilton-Jacobi-Bellman equation, Fokker-Plank equation, dynamic programming

Motivation and Aim: Social networks are a developing information environment. Now the following functions are available on the network: access for informing relatives (Facebook's Safety Check service), publishing data about missing friends (the Google Person Finder service) or promptly informing users of an impending threat and their further actions in case of emergencies (Alerts from Twitter). One of the most effective methods of monitoring and managing above processes is the development and identification of mathematical models that describe the processes of information dissemination in social networks [1]. Such models are described by systems of differential equations, the coefficients of that characterize the distribution of information, population and initial data depend on each information type. To control information in social networks it is necessary to refine the model coefficients and initial data by some additional measurements (the inverse problem) [2].

Methods and Algorithms: One way to solve the problem of improving the coefficients is to reduce the inverse problem to a variational formulation, where the functional satisfies to the Hamilton-Jacobi-Bellman partial differential equation, or characterizes the quadratic deviation of the model data from the experimental ones for systems of stochastic differential equations or partial differential equations. The Tikhonov regularization, gradient methods and genetic algorithm are used for solving ill-posed inverse problems. Results: The reconstructed coefficients, initial data and control functions in mathematical models of social networks (as an example it is considered Digg.com and Twitter [3]) allows one to refine the information dynamic and give the recommendation for control it. Acknowledgements: Supported by the Ministry of Education and Science of Russian Federation and by the President Grant of Russian Federation (No. MK-1214.2017.1).

\section{References}

1. Dai G., Ma R., Wang H., Wang F., Xu K. (2015) Partial differential equations with robin boundary condition in online social networks. Discrete and Continuous Dynamical Systems Series B. 20(6):16091624.

2. Kabanikhin S.I. (2008) Definitions and examples of inverse and ill-posed problem. Journal of Inverse and Ill-Posed Problems. 16(4):317-357.

3. Wang H., Wang F., Xu K. (2013) Modeling information diffusion in online social networks with partial differential equations. arXiv:1310.0505 [cs.SI]. 